\title{
QUASI-TRIVIALITY OF QUANDLES FOR LINK-HOMOTOPY
}

\author{
AYUMU INOUE ${ }^{\dagger}$
}

\begin{abstract}
We introduce the notion of quasi-triviality of quandles and define homology of quasi-trivial quandles. Quandle cocycle invariants are invariant under link-homotopy if they are associated with 2-cocycles of quasi-trivial quandles. We thus obtain a lot of numerical link-homotopy invariants.
\end{abstract}

\section{INTRODUCTION}

Link-homotopy, introduced by Milnor [7], gives rise to an equivalence relation on oriented and ordered links in a 3-sphere. More precisely, two links are said to be link-homotopic if they are related to each other by a finite sequence of ambient isotopies and self-crossing changes, keeping the orientation and ordering. Here, a self-crossing change is a homotopy for a single component of a link, supported in a small ball whose intersection with the component consists of two segments, depicted in Figure 1. The classification problem of links up to link-homotopy is already solved by Habegger and Lin [2] completely. They gave an algorithm which determines whether given links are link-homotopic or not. On the other hand, a table consisting of representatives of all link-homotopy classes is still not known other than partial ones given by Milnor $[7,8]$ for links with 3 or fewer components and by Levine [5] for links with 4 components. We note that both of them utilized numerical invariants to obtain the tables.

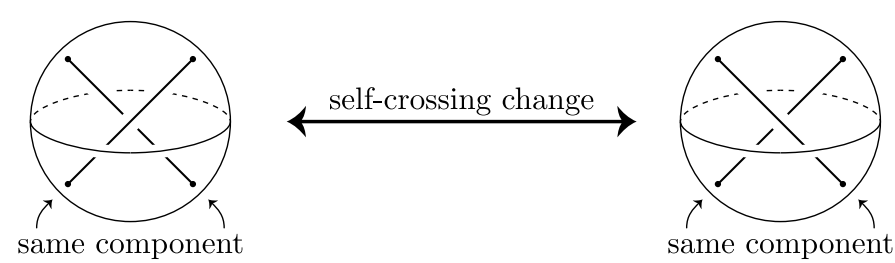

FiguRE 1

A quandle, given by Joyce [4], is an algebraic system consisting of a set together with a binary operation whose definition is strongly motivated in knot theory. Carter et al. [1] introduced quandle homology and gave invariants of links up to ambient isotopy using 2-cocycles of quandles. These invariants, called quandle cocycle invariants, are not invariant under link-homotopy in general. However, in this paper, we introduce the notion of quasi-triviality of quandles and show that

2010 Mathematics Subject Classification. Primary 57M27; Secondary 57M99.

Key words and phrases. link, link-homotopy, quandle, quandle cocycle invariant.

†The author is partially supported by Grant-in-Aid for Research Activity Start-up, No. 23840014, Japan Society for the Promotion of Science. 
quandle cocycle invariants associated with 2-cocycles of quasi-trivial quandles are invariant under link-homotopy (Theorem 3.5), modifying the definition of quandle homology slightly. We thus have a lot of numerical link-homotopy invariants, which might enable us to obtain a table consisting of representatives of all link-homotopy classes. At the end of this paper, as an application of Theorem 3.5, we show a famous fact that the Borromean rings, which is a 3 -component link, is not trivial up to link-homotopy.

Throughout this paper, every link is assumed to be oriented, ordered and in a 3 -sphere. We also assume that every link diagram is oriented and ordered.

\section{Preliminaries}

We devote this section to reviewing a quandle cocycle invariant. Recall that two links are ambient isotopic if and only if their diagrams are related to each other by a finite sequence of Reidemeister moves.

We first review the definition of a quandle. A quandle is a non-empty set $X$ equipped with a binary operation $*: X \times X \rightarrow X$ satisfying the following axioms:

(Q1) For each $x \in X, x * x=x$.

(Q2) For each $x \in X$, a map $* x: X \rightarrow X(\bullet \mapsto \bullet * x)$ is bijective.

(Q3) For each $x, y, z \in X,(x * y) * z=(x * z) *(y * z)$.

The notion of homomorphisms of quandles is appropriately defined. The axioms (Q1), (Q2) and (Q3) of a quandle are closely related to the Reidemeister moves RI, RII and RIII respectively as follows.

An arc coloring of a link diagram $D$ by a quandle $X$ is a map $\{\operatorname{arcs}$ of $D\} \rightarrow X$ satisfying the condition depicted in Figure 2 at each crossing. We call an element of a quandle assigned to an arc by an arc coloring a color of the arc. Suppose $D$ is a link diagram and $D^{\prime}$ a diagram obtained from $D$ by a Reidemeister move. Then, for each arc coloring $\mathscr{A}$ of $D$, we have a unique arc coloring of $D^{\prime}$ whose restriction to arcs unrelated to the deformation coincides with the restriction of $\mathscr{A}$. Indeed, the axioms (Q1), (Q2) and (Q3) of a quandle guarantee that we can perform RI, RII and RIII moves fixing colors of ends respectively (See Figure 3). Therefore, for a fixed quandle, the number of all arc colorings is invariant under ambient isotopy.

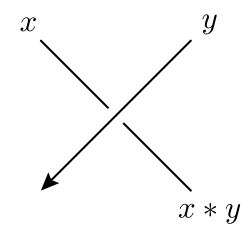

FIGURE 2

We next review quandle homology. Let $X$ be a quandle. Consider the free abelian group $C_{n}^{R}(X)$ generated by all $n$-tuples $\left(x_{1}, x_{2}, \ldots, x_{n}\right) \in X^{n}$ for each $n \geq 1$. We let $C_{0}^{R}(X)=\mathbb{Z}$. Define a map $\partial_{n}: C_{n}^{R} \rightarrow C_{n-1}^{R}$ by

$$
\begin{aligned}
\partial_{n}\left(x_{1}, x_{2}, \ldots, x_{n}\right)=\sum_{i=2}^{n}(-1)^{i}\left\{\left(x_{1}, \ldots, x_{i-1}, x_{i+1}, \ldots, x_{n}\right)\right. & \\
& \left.-\left(x_{1} * x_{i}, \ldots, x_{i-1} * x_{i}, x_{i+1}, \ldots, x_{n}\right)\right\}
\end{aligned}
$$




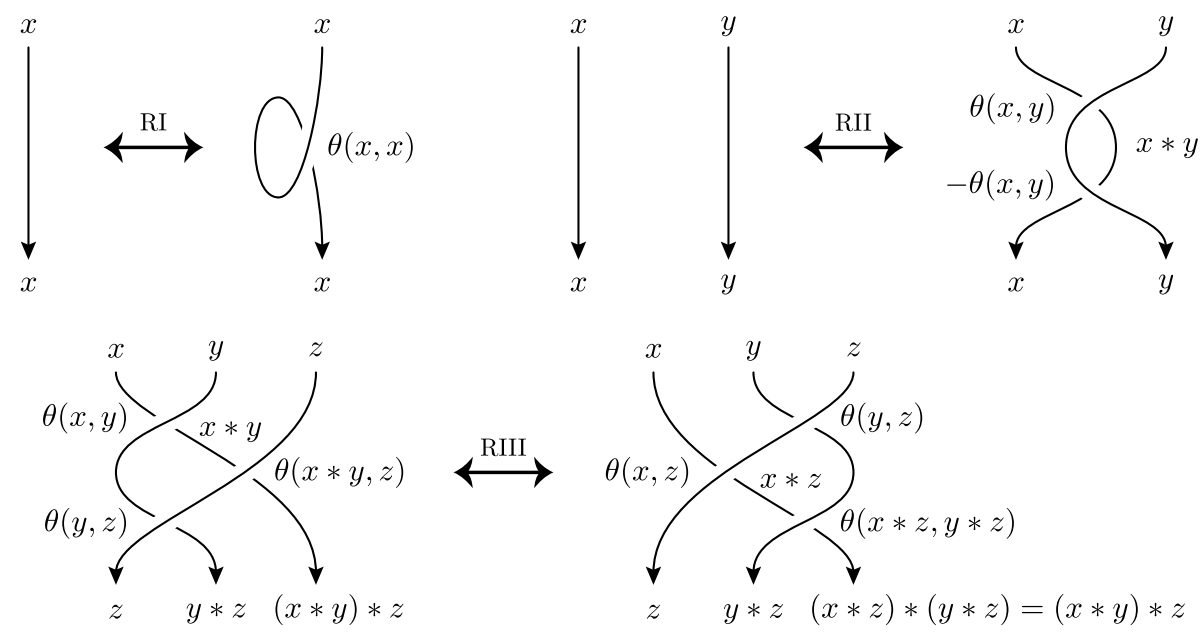

FiguRE 3

for $n \geq 2$, and $\partial_{1}=0$. Then $\partial_{n-1} \circ \partial_{n}=0$. Thus $\left(C_{n}^{R}(X), \partial_{n}\right)$ is a chain complex. Let $C_{n}^{D}(X)$ be a subgroup of $C_{n}^{R}(X)$ generated by $n$-tuples $\left(x_{1}, x_{2}, \ldots, x_{n}\right) \in X^{n}$ with $x_{i}=x_{i+1}$ for some $i$ if $n \geq 2$, and let $C_{n}^{D}(X)=0$ otherwise. It is routine to check that $\partial_{n}\left(C_{n}^{D}(X)\right) \subset C_{n-1}^{D}(X)$. Therefore, putting $C_{n}^{Q}(X)=C_{n}^{R}(X) / C_{n}^{D}(X)$, we have a chain complex $\left(C_{n}^{Q}(X), \partial_{n}\right)$. Let $A$ be an abelian group. The $n$-th quandle homology group $H_{n}^{Q}(X ; A)$ with coefficients in $A$ is the $n$-th homology group of the chain complex $\left(C_{n}^{Q}(X) \otimes A, \partial_{n} \otimes\right.$ id). The $n$-th quandle cohomology group $H_{Q}^{n}(X ; A)$ with coefficients in $A$ is the $n$-th cohomology group of the cochain complex $\left(\operatorname{Hom}\left(C_{n}^{Q}(X), A\right), \operatorname{Hom}\left(\partial_{n}\right.\right.$, id $\left.)\right)$. We note that $\theta \in \operatorname{Hom}\left(C_{2}^{Q}(X), A\right)$ is a 2-cocycle if and only if $\theta$ satisfies the following conditions:

(C1) For each $x \in X, \theta(x, x)=0$.

(C2) For each $x, y, z \in X, \theta(\partial(x, y, z))=0$, that is, $\theta(x, y)+\theta(x * y, z)=\theta(x, z)+\theta(x * z, y * z)$.

Associated with a 2-cocycle of a quandle, we can define an weight of an arc coloring as follows. Suppose $X$ is a quandle and $\theta \in \operatorname{Hom}\left(C_{2}^{Q}(X), A\right)$ a 2-cocycle with coefficients in an abelian group $A$. The $i$-th weight of an arc coloring $\mathscr{A}$ of a link diagram $D$ by $X$ associated with $\theta$ is a value

$$
W(\mathscr{A}, \theta ; i)=\sum \varepsilon \cdot \theta(x, y) \in A,
$$

where the sum runs over the crossings of $D$, each of which consists of under arcs belonging to the $i$-th component and an over arc, $\varepsilon$ is 1 or -1 depending on whether the crossing is positive or negative respectively, and $x, y \in X$ denote colors of arcs around the crossing as depicted in Figure 2. We have the following theorem.

Theorem 2.1 (Carter et al. [1]). Let $X$ be a quandle and $\theta \in \operatorname{Hom}\left(C_{2}^{Q}(X), A\right)$ a 2-cocycle with coefficients in an abelian group $A$. Then an weight of an arc coloring by $X$ associated with $\theta$ is invariant under Reidemeister moves.

Proof. Let $\mathscr{A}$ be an arc coloring of a link diagram by $X$. Consider to perform a Reidemeister move for this colored diagram. An RI move for a segment of the $i$-th component only adds or subtracts $\theta(x, x)$ to or from $W(\mathscr{A}, \theta ; i)$ with some $x \in X$ 
(See the upper left of Figure 3$)$. It does not change $W(\mathscr{A}, \theta ; i)$ because $\theta$ satisfies the condition ( $\mathrm{C} 1)$. An RII move, by which a segment of the $i$-th component passes under a some arc, only adds or subtracts $\theta(x, y)-\theta(x, y)=0$ to or from $W(\mathscr{A}, \theta ; i)$ with some $x, y \in X$ (See the upper right of Figure 3 ). An RIII move adds $\pm \theta(\partial(x, y, z))$ to $W(\mathscr{A}, \theta ; i)$ with some $x, y, z \in X$ if the innermost arcs related to the deformation belong to the $i$-th component (See the bottom of Figure 3 ). Since $\theta$ satisfies the condition $(\mathrm{C} 2)$, it does not change $W(\mathscr{A}, \theta ; i)$.

Suppose again that $X$ is a quandle and $\theta \in \operatorname{Hom}\left(C_{2}^{Q}(X), A\right)$ a 2-cocycle with coefficients in an abelian group $A$. Theorem 2.1 says that, for each link $L$ and index $i$, the multiset $\Phi(L, \theta ; i)$ consisting of $i$-th weights of all arc coloring of a diagram of $L$ by $X$ associated with $\theta$ does not depend on the choice of the diagram. Thus $\Phi(L, \theta ; i)$ is invariant under ambient isotopy. The $i$-th quandle cocycle invariant of $L$ associated with $\theta$ is this multiset $\Phi(L, \theta ; i)$.

\section{QUASI-TRIVIAL QUANDLE AND LINK-HOMOTOPY}

Although the number of all arc colorings is invariant under ambient isotopy, it is not invariant under self-crossing changes in general. Indeed, the trefoil knot is deformed into the unknot by a self-crossing change, but they are distinguished by the numbers of all arc colorings. Thus a quandle cocycle invariant is not invariant under link-homotopy in general. On the other hand, in this section, we show that a self-crossing change on a diagram also relates arc colorings of the original and deformed diagrams uniquely if we use a certain quandle, named a quasi-trivial quandle, for arc colorings. Since two links are link-homotopic if and only if their diagrams are related to each other by a finite sequence of Reidemeister moves and self-crossing changes on diagrams, the number of all arc colorings by a quasi-trivial quandle is invariant under link-homotopy. Furthermore, modifying the definition of quandle homology slightly, we will have a quandle cocycle invariant which is invariant under link-homotopy.

To define a quasi-trivial quandle, we first review the automorphism group and inner automorphism group of a quandle. The automorphism group of a quandle $X$ is a group $\operatorname{Aut}(X)$ consisting of all automorphisms of $X$ together with a product given by the composition of maps. The axioms (Q2) and (Q3) of a quandle guarantee that, for each $x \in X$, the bijection $* x: X \rightarrow X$ is an automorphism of $X$. The inner automorphism group $\operatorname{Inn}(X)$ is the subgroup of $\operatorname{Aut}(X)$ generated by all automorphisms $* x: X \rightarrow X$. We call an element of $\operatorname{Inn}(X)$ an inner automorphism of $X$.

Definition 3.1. A quandle $X$ is said to be quasi-trivial if $x * \varphi(x)=x$ for each $x \in X$ and $\varphi \in \operatorname{Inn}(X)$.

As mentioned above, we have the following proposition.

Proposition 3.2. Let $X$ be a quasi-trivial quandle. Suppose $D$ is a link diagram and $D^{\prime}$ a diagram obtained from $D$ by a self-crossing change. Then, for each arc coloring $\mathscr{A}$ of $D$ by $X$, we have a unique arc coloring of $D^{\prime}$ whose restriction to arcs unrelated to the deformation coincides with the restriction of $\mathscr{A}$.

Proof. Assume that the self-crossing change is performed at a crossing $c$. Let $x, y, x * y \in X$ denote colors of $\operatorname{arcs}$ around $c$ by $\mathscr{A}$ as depicted in Figure 2. Since 

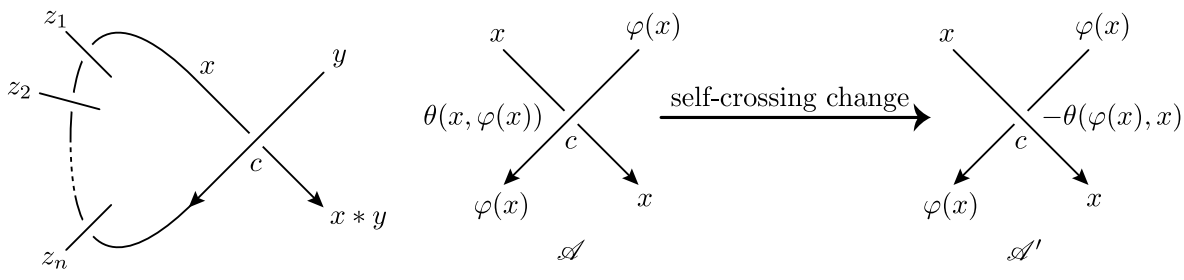

FigURE 4

under arcs and an over arc around $c$ belong to the same component, we have

$$
y=\left(* z_{n}\right)^{\varepsilon_{n}} \circ \cdots \circ\left(* z_{2}\right)^{\varepsilon_{2}} \circ\left(* z_{1}\right)^{\varepsilon_{1}}(x)
$$

with some $n \in \mathbb{Z}_{\geq 0}, z_{i} \in X$ and $\varepsilon_{i} \in\{ \pm 1\}$ (See the left-hand side of Figure 4). Let $\varphi \in \operatorname{Inn}(X)$ denote the inner automorphism $\left(* z_{n}\right)^{\varepsilon_{n}} \circ \cdots \circ\left(* z_{2}\right)^{\varepsilon_{2}} \circ\left(* z_{1}\right)^{\varepsilon_{1}}$. By the assumption that $X$ is quasi-trivial, $x * y=x * \varphi(x)=x$. Thus, remarking that $\varphi(x) * x=\varphi(x)$, we have a unique arc coloring $\mathscr{A}^{\prime}$ of $D^{\prime}$ whose restriction to arcs unrelated to the deformation coincides with the restriction of $\mathscr{A}$ (See the right-hand side of Figure 4).

Remark 3.3. For a link $L$, we have the knot quandle $Q(L)$, defined by Joyce [4], which is invariant under ambient isotopy (The same notion is given by Matveev [6]). An arc coloring of a diagram of $L$ by a quandle $X$ is just a diagrammatic presentation of a homomorphism $Q(L) \rightarrow X$.

We further have the reduced knot quandle $R Q(L)$ of $L$, given by Hughes [3], which is the quasi-trivialization of $Q(L)$. Suppose $X$ is a quasi-trivial quandle. Then each homomorphism $Q(L) \rightarrow X$ factors through a homomorphism $R Q(L) \rightarrow X$. Conversely, each homomorphism $R Q(L) \rightarrow X$ uniquely lifts to a homomorphism $Q(L) \rightarrow X$. Thus an arc coloring of a diagram of $L$ by $X$ is just a diagrammatic presentation of a homomorphism $R Q(L) \rightarrow X$. Hughes showed that $R Q(L)$ is invariant under link-homotopy. It gives an alternative proof for Proposition 3.2.

Proposition 3.2 says that, for a fixed quasi-trivial quandle, the number of all arc colorings is invariant under link-homotopy. However, weights of arc colorings are not invariant under self-crossing changes in general, even if we use quasi-trivial quandles for arc colorings. We thus have to modify the definition of quandle homology slightly, as follows, so that weights are invariant under self-crossing changes.

Let $X$ be a quasi-trivial quandle. Define the free abelian groups $C_{n}^{R}(X)$ and boundary maps $\partial_{n}: C_{n}^{R}(X) \rightarrow C_{n-1}^{R}(X)$ as in Section 2. Let $C_{n}^{D, q t}(X)$ be a subgroup of $C_{n}^{R}(X)$ generated by $n$-tuples $\left(x_{1}, x_{2}, \ldots, x_{n}\right) \in X^{n}$ with $x_{i}=x_{i+1}$ for some $i$ and $n$-tuples $\left(x_{1}, \varphi\left(x_{1}\right), x_{3}, \ldots, x_{n}\right) \in X^{n}$ for some $\varphi \in \operatorname{Inn}(X)$ if $n \geq 2$. Let $C_{n}^{D, q t}(X)=0$ if $n=0,1$. It is easy to see that $\partial_{n}\left(C_{n}^{D, q t}(X)\right) \subset C_{n-1}^{D, q t}(X)$. Thus, putting $C_{n}^{Q, q t}(X)=C_{n}^{R}(X) / C_{n}^{D, q t}(X)$, we have a chain complex $\left(C_{n}^{Q, q t}(X), \partial_{n}\right)$.

Suppose that $A$ is an abelian group. The $n$-th quasi-trivial quandle homology group $H_{n}^{Q, q t}(X ; A)$ with coefficients in $A$ is the $n$-th homology group of the chain complex $\left(C_{n}^{Q, q t}(X) \otimes A, \partial_{n} \otimes \mathrm{id}\right)$. The $n$-th quasi-trivial quandle cohomology group $H_{Q, q t}^{n}(X ; A)$ with coefficients in $A$ is the $n$-th cohomology group of the cochain complex $\left(\operatorname{Hom}\left(C_{n}^{Q, q t}(X), A\right), \operatorname{Hom}\left(\partial_{n}\right.\right.$, id $\left.)\right)$. We note that $\theta \in \operatorname{Hom}\left(C_{2}^{Q, q t}(X), A\right)$ is 
a 2-cocycle if and only if $\theta$ satisfies the conditions (C1), (C2) in Section 2 and the following condition:

(C3) For each $x \in X$ and $\varphi \in \operatorname{Inn}(X), \theta(x, \varphi(x))=0$.

Proposition 3.4. Let $X$ be a quasi-trivial quandle and $\theta \in \operatorname{Hom}\left(C_{2}^{Q, q t}(X), A\right)$ a 2-cocycle with coefficients in an abelian group $A$. Then an weight of an arc coloring by $X$ associated with $\theta$ is invariant under self-crossing changes on diagrams.

Proof. Let $\mathscr{A}$ be an arc coloring of a link diagram by $X$. A self-crossing change at a crossing of the diagram subtracts $\pm(\theta(x, \varphi(x))+\theta(\varphi(x), x))$ from $W(\mathscr{A}, \theta ; i)$ with some $x \in X$ and $\varphi \in \operatorname{Inn}(X)$ if the crossing consists of arcs belonging to the $i$-th component (See the right-hand side of Figure 4 ). Since $\theta$ satisfies the condition (C3), it does not change $W(\mathscr{A}, \theta ; i)$.

By Theorem 2.1 and Proposition 3.4, we have the following theorem immediately.

Theorem 3.5. Let $X$ be a quasi-trivial quandle and $\theta \in \operatorname{Hom}\left(C_{2}^{Q, q t}(X), A\right)$ be a 2-cocycle with coefficients in an abelian group $A$. Then, for each link $L$ and index $i$, the $i$-th quandle cocycle invariant $\Phi(L, \theta ; i)$ is invariant under link-homotopy.

Remark 3.6. A trivial quandle is a non-empty finite set $X$ equipped with a binary operation $*$ satisfying $x * y=x$ for each $x, y \in X$. Carter et al. [1] showed that, for each 2-cocycle $\theta \in \operatorname{Hom}\left(C_{2}^{Q}(X), A\right)$ with coefficients in an abelian group $A$ and index $i$, the $i$-th quandle cocycle invariant $\Phi(L, \theta ; i)$ is completely determined by the linking numbers of $L$. Since the linking numbers are invariant under link-homotopy, $\Phi(L, \theta ; i)$ is also invariant under link-homotopy. It is easy to see that each trivial quandle is quasi-trivial and its 2-cocycle satisfies not only the conditions (C1) and (C2) but the condition (C3).

\section{EXAMPLE}

In this final section, as an application of Theorem 3.5, we show a famous fact that the Borromean rings is not trivial up to link-homotopy.

Let $X$ be a set consisting of twelve elements $a_{i}, b_{i}$ and $c_{i}(1 \leq i \leq 4)$. Define a binary operation $*$ on $X$ by Table 1 whose $(i+1, j+1)$-entry denotes $x * y$ with $x$ being the $(i+1,1)$-entry and $y$ the $(1, j+1)$-entry. Then $X$ with $*$ is in fact a quasi-trivial quandle. Furthermore, define an element $\theta \in \operatorname{Hom}\left(C_{2}^{Q, q t}(X), \mathbb{Z}_{2}\right)$ by Table 2 whose $(i+1, j+1)$-entry denotes $\theta(x, y)$ with $x$ being the $(i+1,1)$-entry and $y$ the $(1, j+1)$-entry. It is routine to check that $\theta$ is a 2 -cocycle.

Suppose $D_{T}$ and $D_{B}$ are diagrams of the trivial 3-component link $L_{T}$ and the Borromean rings $L_{B}$ depicted in Figure 5 respectively. Since $D_{T}$ has no crossing, for each arc coloring $\mathscr{A}_{T}$ of $D_{T}$ by $X$ and index $i, W\left(\mathscr{A}_{T}, \theta ; i\right)$ is always equal to 0 . On the other hand, we have an arc coloring $\mathscr{A}_{B}$ of $D_{B}$ by $X$, depicted in Figure 5 , satisfying $W\left(\mathscr{A}_{B}, \theta ; i\right)=1$ for each index $i$. It says that $\Phi\left(L_{T}, \theta ; i\right) \neq \Phi\left(L_{B}, \theta ; i\right)$ for each index $i$. Thus, by Theorem 3.5, the Borromean rings $L_{B}$ is not link-homotopic to the trivial 3-component link $L_{T}$.

Remark 4.1. Since the number of all arc colorings of $D_{B}$ by $X$ is equal to the number for $D_{T}$, we essentially have to compute the quandle cocycle invariants to distinguish $L_{B}$ and $L_{T}$ up to link-homotopy. 


\begin{tabular}{|c||c|c|c|c|c|c|c|c|c|c|c|c|}
\hline$*$ & $a_{1}$ & $a_{2}$ & $a_{3}$ & $a_{4}$ & $b_{1}$ & $b_{2}$ & $b_{3}$ & $b_{4}$ & $c_{1}$ & $c_{2}$ & $c_{3}$ & $c_{4}$ \\
\hline \hline$a_{1}$ & $a_{1}$ & $a_{1}$ & $a_{1}$ & $a_{1}$ & $a_{2}$ & $a_{2}$ & $a_{2}$ & $a_{2}$ & $a_{3}$ & $a_{3}$ & $a_{3}$ & $a_{3}$ \\
\hline$a_{2}$ & $a_{2}$ & $a_{2}$ & $a_{2}$ & $a_{2}$ & $a_{1}$ & $a_{1}$ & $a_{1}$ & $a_{1}$ & $a_{4}$ & $a_{4}$ & $a_{4}$ & $a_{4}$ \\
\hline$a_{3}$ & $a_{3}$ & $a_{3}$ & $a_{3}$ & $a_{3}$ & $a_{4}$ & $a_{4}$ & $a_{4}$ & $a_{4}$ & $a_{1}$ & $a_{1}$ & $a_{1}$ & $a_{1}$ \\
\hline$a_{4}$ & $a_{4}$ & $a_{4}$ & $a_{4}$ & $a_{4}$ & $a_{3}$ & $a_{3}$ & $a_{3}$ & $a_{3}$ & $a_{2}$ & $a_{2}$ & $a_{2}$ & $a_{2}$ \\
\hline$b_{1}$ & $b_{3}$ & $b_{3}$ & $b_{3}$ & $b_{3}$ & $b_{1}$ & $b_{1}$ & $b_{1}$ & $b_{1}$ & $b_{2}$ & $b_{2}$ & $b_{2}$ & $b_{2}$ \\
\hline$b_{2}$ & $b_{4}$ & $b_{4}$ & $b_{4}$ & $b_{4}$ & $b_{2}$ & $b_{2}$ & $b_{2}$ & $b_{2}$ & $b_{1}$ & $b_{1}$ & $b_{1}$ & $b_{1}$ \\
\hline$b_{3}$ & $b_{1}$ & $b_{1}$ & $b_{1}$ & $b_{1}$ & $b_{3}$ & $b_{3}$ & $b_{3}$ & $b_{3}$ & $b_{4}$ & $b_{4}$ & $b_{4}$ & $b_{4}$ \\
\hline$b_{4}$ & $b_{2}$ & $b_{2}$ & $b_{2}$ & $b_{2}$ & $b_{4}$ & $b_{4}$ & $b_{4}$ & $b_{4}$ & $b_{3}$ & $b_{3}$ & $b_{3}$ & $b_{3}$ \\
\hline$c_{1}$ & $c_{2}$ & $c_{2}$ & $c_{2}$ & $c_{2}$ & $c_{3}$ & $c_{3}$ & $c_{3}$ & $c_{3}$ & $c_{1}$ & $c_{1}$ & $c_{1}$ & $c_{1}$ \\
\hline$c_{2}$ & $c_{1}$ & $c_{1}$ & $c_{1}$ & $c_{1}$ & $c_{4}$ & $c_{4}$ & $c_{4}$ & $c_{4}$ & $c_{2}$ & $c_{2}$ & $c_{2}$ & $c_{2}$ \\
\hline$c_{3}$ & $c_{4}$ & $c_{4}$ & $c_{4}$ & $c_{4}$ & $c_{1}$ & $c_{1}$ & $c_{1}$ & $c_{1}$ & $c_{3}$ & $c_{3}$ & $c_{3}$ & $c_{3}$ \\
\hline$c_{4}$ & $c_{3}$ & $c_{3}$ & $c_{3}$ & $c_{3}$ & $c_{2}$ & $c_{2}$ & $c_{2}$ & $c_{2}$ & $c_{4}$ & $c_{4}$ & $c_{4}$ & $c_{4}$ \\
\hline
\end{tabular}

TABle 1

\begin{tabular}{|c||c|c|c|c|c|c|c|c|c|c|c|c|}
\hline$\theta$ & $a_{1}$ & $a_{2}$ & $a_{3}$ & $a_{4}$ & $b_{1}$ & $b_{2}$ & $b_{3}$ & $b_{4}$ & $c_{1}$ & $c_{2}$ & $c_{3}$ & $c_{4}$ \\
\hline \hline$a_{1}$ & 0 & 0 & 0 & 0 & 1 & 0 & 1 & 0 & 1 & 1 & 0 & 0 \\
\hline$a_{2}$ & 0 & 0 & 0 & 0 & 0 & 1 & 0 & 1 & 0 & 0 & 1 & 1 \\
\hline$a_{3}$ & 0 & 0 & 0 & 0 & 1 & 0 & 1 & 0 & 1 & 1 & 0 & 0 \\
\hline$a_{4}$ & 0 & 0 & 0 & 0 & 0 & 1 & 0 & 1 & 0 & 0 & 1 & 1 \\
\hline$b_{1}$ & 1 & 1 & 0 & 0 & 0 & 0 & 0 & 0 & 1 & 0 & 1 & 0 \\
\hline$b_{2}$ & 0 & 0 & 1 & 1 & 0 & 0 & 0 & 0 & 0 & 1 & 0 & 1 \\
\hline$b_{3}$ & 1 & 1 & 0 & 0 & 0 & 0 & 0 & 0 & 1 & 0 & 1 & 0 \\
\hline$b_{4}$ & 0 & 0 & 1 & 1 & 0 & 0 & 0 & 0 & 0 & 1 & 0 & 1 \\
\hline$c_{1}$ & 1 & 0 & 1 & 0 & 1 & 1 & 0 & 0 & 0 & 0 & 0 & 0 \\
\hline$c_{2}$ & 0 & 1 & 0 & 1 & 0 & 0 & 1 & 1 & 0 & 0 & 0 & 0 \\
\hline$c_{3}$ & 1 & 0 & 1 & 0 & 1 & 1 & 0 & 0 & 0 & 0 & 0 & 0 \\
\hline$c_{4}$ & 0 & 1 & 0 & 1 & 0 & 0 & 1 & 1 & 0 & 0 & 0 & 0 \\
\hline
\end{tabular}

TABLE 2

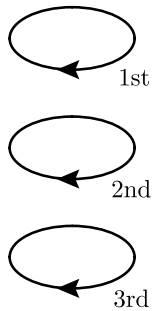

$D_{T}$

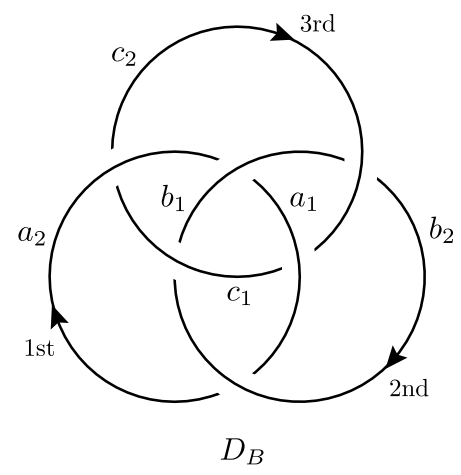

Figure 5 


\section{Appendix. Decomposition of Quandle Cocycle invariant}

In this appendix, we see that each quandle cocycle invariant can be decomposed into multisets which are still invariant under ambient isotopy or link-homotopy. It seems that these multisets are more useful than a quandle cocycle invariant itself to classify links.

We first note that the inner automorphism group of a quandle naturally acts on the quandle. Therefore each quandle is decomposed into the orbits of the inner automorphism group.

Let $X$ be a quandle and $\cup_{\lambda \in \Lambda} X_{\lambda}$ the orbit decomposition of $X$. Remark that each arc coloring of a link diagram maps arcs belonging to the same component in the same orbit. We thus call an arc coloring of a diagram of an $n$-component link by $X$ to be in $\left(X_{\lambda_{1}}, X_{\lambda_{2}}, \ldots, X_{\lambda_{n}}\right)$ if it maps arcs belonging to the $i$-th component in $X_{\lambda_{i}}$. Obviously, the number of all arc colorings in $\left(X_{\lambda_{1}}, X_{\lambda_{2}}, \ldots, X_{\lambda_{n}}\right)$ is invariant under ambient isotopy for each $n$-tuple $\left(\lambda_{1}, \lambda_{2}, \ldots, \lambda_{n}\right) \in \Lambda^{n}$.

Let $\theta \in \operatorname{Hom}\left(C_{2}^{Q}(X), A\right)$ be a 2-cocycle with coefficients in an abelian group $A$. For an $n$-component link $L$ and $n$-tuple $\left(\lambda_{1}, \lambda_{2}, \ldots, \lambda_{n}\right) \in \Lambda^{n}$, consider the multiset $\Phi\left(L, \theta ; X_{\lambda_{1}}, X_{\lambda_{2}}, \ldots, X_{\lambda_{n}} ; i\right)$ consisting of $i$-th weights of all arc colorings of a diagram of $L$ in $\left(X_{\lambda_{1}}, X_{\lambda_{2}}, \ldots, X_{\lambda_{n}}\right)$. Then obviously

$$
\Phi(L, \theta ; i)=\bigcup_{\left(\lambda_{1}, \lambda_{2}, \ldots, \lambda_{n}\right) \in \Lambda^{n}} \Phi\left(L, \theta ; X_{\lambda_{1}}, X_{\lambda_{2}}, \ldots, X_{\lambda_{n}} ; i\right)
$$

and each $\Phi\left(L, \theta ; X_{\lambda_{1}}, X_{\lambda_{2}}, \ldots, X_{\lambda_{n}} ; i\right)$ is invariant under ambient isotopy.

Of course, the above arguments hold even if $X$ is a quasi-trivial quandle and $\theta \in \operatorname{Hom}\left(C_{2}^{Q, q t}(X), A\right)$ a 2 -cocycle. In this case, the number of all arc colorings in $\left(X_{\lambda_{1}}, X_{\lambda_{2}}, \ldots, X_{\lambda_{n}}\right)$ and the multiset $\Phi\left(L, \theta ; X_{\lambda_{1}}, X_{\lambda_{2}}, \ldots, X_{\lambda_{n}} ; i\right)$ are invariant under link-homotopy.

\section{REFERENCES}

1. J. S. Carter, D. Jelsovsky, S. Kamada, L. Langford and M. Saito, Quandle cohomology and state-sum invariants of knotted curves and surfaces, Trans. Amer. Math. Soc. 355 (2003), no. 10, 3947-3989.

2. N. Habegger and X. S. Lin, The classification of links up to link-homotopy, J. Amer. Math. Soc. 3 (1990), no. 2, 389-419.

3. J. R. Hughes, Link homotopy invariant quandles, J. Knot Theory Ramifications 20 (2011), no. $5,763-773$.

4. D. Joyce, A classifying invariant of knots, the knot quandle, J. Pure Appl. Algebra 23 (1982), no. $1,37-65$.

5. J. P. Levine, An approach to homotopy classification of links, Trans. Amer. Math. Soc. 306 (1988), no. 1, 361-387.

6. S. V. Matveev, Distributive groupoids in knot theory, Mat. Sb. (N.S.) 119 (161) (1982), 78-88 (English translation: Math. USSR-Sb. 47 (1984), 73-83).

7. J. Milnor, Link groups, Ann. of Math. 59 (1954), 177-195.

8. J. Milnor, Isotopy of links, Algebraic geometry and topology (A symposium in honor of S. Lefschetz), Princeton Univ. Press, Princeton, N. J., 1957, 280-306.

Department of Mathematical and Computing Sciences, Tokyo Institute of Technology, Ookayama, Meguro-Ku, Tokyo, 152-8552 Japan

E-mail address: ayumu.inoue@math.titech.ac.jp 